\title{
Electroacupuncture for Functional Constipation: A Multicenter, Randomized, Control Trial
}

\author{
Xiao Wu, ${ }^{1}$ Cuihong Zheng, ${ }^{1}$ Xiaohu Xu, ${ }^{2}$ Pei Ding, ${ }^{1}$ Fan Xiong, \\ Man Tian, ${ }^{1}$ Ying Wang, ${ }^{1}$ Haoxu Dong, ${ }^{1}$ Mingmin Zhang, ${ }^{2}$ Wei Wang, \\ Shabei $\mathrm{Xu},{ }^{3}$ Minjie Xie, ${ }^{3}$ and Guangying Huang ${ }^{1}$ \\ ${ }^{1}$ Institute of Integrated Traditional Chinese and Western Medicine, Tongji Hospital, Tongji Medical College, \\ Huazhong University of Science and Technology, 1095 Jiefang Avenue, Wuhan, Hubei 430030, China \\ ${ }^{2}$ Department of Integrated Traditional Chinese and Western Medicine, Tongji Hospital, Tongji Medical College, \\ Huazhong University of Science and Technology, 1095 Jiefang Avenue, Wuhan, Hubei 430030, China \\ ${ }^{3}$ Department of Neurology, Tongji Hospital, Tongji Medical College, Huazhong University of Science and Technology, \\ 1095 Jiefang Avenue, Wuhan, Hubei 430030, China
}

Correspondence should be addressed to Guangying Huang; gyhuang@tjh.tjmu.edu.cn

Received 26 August 2016; Revised 26 October 2016; Accepted 30 November 2016; Published 31 January 2017

Academic Editor: Karen Nieber

Copyright (C) 2017 Xiao Wu et al. This is an open access article distributed under the Creative Commons Attribution License, which permits unrestricted use, distribution, and reproduction in any medium, provided the original work is properly cited.

\begin{abstract}
Background and Aim. To investigate the efficacy and safety of electroacupuncture (EA) with different current intensities for functional constipation (FC) and to assess whether the effects of EA with different current intensities are superior to the mosapride. Methods. Patients with FC were randomly divided into low current intensity group (LCI), high current intensity group (HCI), and mosapride group (MC). The primary outcome was three or more spontaneous bowel movements (SBMs) per week and an increase of one or more SBMs from baseline during at least 3 of the 4 weeks. Results. The primary outcome was reached by $53.45 \%, 66.15 \%$, and $52.24 \%$ of the patients who received LCI, HCI, and mosapride, respectively. EA can significantly improve the weekly SBMs and stool consistency and reduce straining severity $(p<0.0001$, all). HCI improved the quality of life better than mosapride $(p<0.05)$ and reduced the proportion of severe constipation more than LCI and mosapride ( $p<0.05$, both). Conclusions. EA is effective and safe at both current intensities for FC; therapeutic effects of LCI and HCI are not superior to mosapride. EA is superior to mosapride in improving patients' life quality and satisfaction level of treatment; EA has fewer adverse events than mosapride.
\end{abstract}

\section{Introduction}

Functional constipation (FC) is a common type of functional gastrointestinal disorders (FGIDs) [1]. Generally, constipation is defined as infrequent bowel movements (BMs), typically fewer than 3 times per week; patients have a more extensive set of symptoms, including hard or lumpy stools, straining, and a sensation of incomplete rectal evacuation, abdominal discomfort, bloating, a sense of anorectal blockage during defecation, and the need for manual maneuvers during defecation [2]. Symptoms of FC are extremely common that can negatively affect the quality of patients' life [3, 4]. Because of its high prevalence rate [5] and the severe symptoms, this disorder represents a large economic burden to the health care system $[3,6,7]$. Even though there are multiple medications (including prescription laxatives, overthe-counter products, and fibre supplementation) for chronic constipation, the level of satisfaction with treatments is still poor $[4,8]$. Therefore, many patients turn to choose complementary and alternative options from traditional Chinese medicine (TCM) for their constipation symptoms [9].

Acupuncture is a crucial part of TCM, with thousands of years' history. Because of its convenience, safety, and unique therapeutic effects, acupuncture has gained increasing popularity in Western countries [10]. Electroacupuncture (EA), a modified procedure with acupuncture and electrical current stimulation, has been widely used in recent years [11] due to the convenience of controlling and regulating parameters of current stimulation. Numerous studies have 
been performed to investigate that acupuncture or EA can alter gastrointestinal motility functions and benefit patients with functional gastrointestinal diseases [12, 13]. According to a review [14], the most popular acupoints for FC are ST25 (Tianshu), ST37 (Shangjuxu), BL25 (Dachangshu), ST36 (Zusanli), and TE6 (Zhigou). Various acupoint groups have been used for constipation: abdomen acupoints (e.g., ST25) plus crus acupoints (e.g., ST36, ST37) or forearm acupoints (e.g., TE6, LI11) are the most common combinations. Studies use ST37 (Shangjuxu) combined with LI11 (Quchi) for functional constipation patients are infrequent. Different stimulation frequencies are used in these studies, including $2 \mathrm{~Hz} / 200 \mathrm{~Hz}$ [15], $2 \mathrm{~Hz} / 100 \mathrm{~Hz}$ [16], $2 \mathrm{~Hz} / 15 \mathrm{~Hz}$ [17, 18], $2 \mathrm{~Hz} / 10 \mathrm{~Hz}$ [19], and $10 \mathrm{~Hz} / 50 \mathrm{~Hz}$ [20]. The frequency of $2 / 50 \mathrm{~Hz}$ is rarely used. Trials conducted with low current intensity and high current intensity of EA for functional constipation are especially rare.

For these reasons, we designed a multicenter, randomized, parallel, controlled trial using acupoints LI11 plus ST37 with a frequency of $2 / 50 \mathrm{~Hz}$ for functional constipation. In this study, our aims were (i) to evaluate the efficacy and safety of EA with different current intensities for patients with FC and (ii) to assess whether the effects of EA with different current intensities are superior to the mosapride.

\section{Patients and Methods}

2.1. Study Design. The total study period was 9 weeks, including 1-week baseline, 4 -week treatment, and 4-week follow-up. The trial was conducted in accordance with the protocol [21], which is available with the full text at http:// trialsjournal.biomedcentral.com/. This study was approved by the Clinical Trial Ethics Committee of Tongji Medical College, Huazhong University of Science and Technology (approval number FWA00007304), and was conducted in accordance with the provisions of the Declaration of Helsinki and Good Clinical Practice guidelines. This study was registered on the Clinical Trials system (ClinicalTrials.gov ID: NCT01274793).

2.2. Patients. Patients with FC (the diagnostic criteria were based on Rome III Criteria [22]) were recruited from the following three hospitals from December 14, 2011 (first patient enrolled), to March 29, 2015 (last patient completed): Affiliated Tongji Hospital of Huazhong University of Science and Technology, Campus Hospital of Huazhong University of Science and Technology, and Affiliated Hubei Provincial Hospital of Hubei University of TCM.

2.2.1. Inclusion Criteria. Patients were included if they met all of the following criteria: (i) diagnosis of functional constipation according to the Rome III Criteria; (ii) aged between 18 and 70 years old (the range of age initially was from 18 to 65 years; during the trial, many patients aged older than 65 years were strongly willing to participant in the trial, and they met all the other inclusion criteria. Participants in some trials [23-25] conducted for constipation were older than 80 years. The protocol was immediately amended in accordance with that recommendation, the age of participants were expanded to not older than 70 years); (iii) not taking any drugs that promote gastrointestinal movements at least during the 1 week prior to randomization; (iv) willing to sign the informed consent form before randomization.

2.2.2. Exclusion Criteria. The exclusion criteria were as follows: (i) unconsciousness, psychosis, or failure to express subjective symptoms; (ii) being complicated with serious cardiovascular, hepatic, or renal diseases, or bleeding disorders; (iii) being combined with progressing malignancy or other serious debilitating illnesses; (iv) women in gestation or lactation periods.

Before randomization, all patients were (i) asked to discontinue any medications for constipation symptoms (e.g., anticholinergic agents, narcotics, and laxatives) (but glycerine enema up to $10 \mathrm{~mL}$ daily was allowed as a rescue medication if a patient did not have defecation for three or more consecutive days; the details about the use of rescue medication were recorded in patient diaries) and (ii) fully informed and asked to sign a written informed consent based on their own will.

During an initial screening period, all patients received routine tests of blood, urine, stool, and blood biochemical (ALT, AST, BUN, and Scr), electrocardiogram (ECG), and a colonoscopy prior to randomization. These tests would help identify and exclude patients who have serious heart, liver, kidney, or other severe diseases, and a colonoscopy would exclude organic diseases. To exclude pregnant women, urine HCG or blood $\beta$-HCG were tested for possible pregnancy. All the patients received routine tests of blood, urine, and stool after completing the treatment, which would help assess the adverse events.

All patients were required to record defecation diaries, including the times of spontaneous bowel movements (SBMs) per day, stool consistency, and severity of straining. The evaluation criteria of stool consistency was based on the Bristol Stool Form Scale (BSFS) [26], which ranges from 1 to 7 , with lower scores indicating harder stool and higher scores indicating more liquid stool. Scores for straining severity range from 0 to 3,0 indicating not at all, 1 a little bit, 2 a moderate amount, and 3 a great deal and an extreme amount. This course would be continuing to 8 weeks.

2.3. Randomization and Blinding. Patients were completely randomized into the low current intensity group (LCI), high current intensity group (HCI), and mosapride citrate tablet control group (MC) at a ratio of $1: 1: 1$. We used R2.0 software to generate the randomization sequence. The designated researchers prepared the sequence through the use of sealed, opaque, sequentially numbered envelopes. One person at each hospital was responsible for the envelopes. To preserve masking, only the acupuncturists knew the treatment allocation. The patients and recruiters were all unaware of study-group assignments. Blinded evaluation (the curative effect was evaluated by a third party who did not know the assignment) and blinded statistical analysis were emphasized during the data collection and analysis stage.

Doctors from gastroenterology department of the local hospitals were invited to screen participants. All the licensed 
acupuncturists were experienced and had years of clinical training. And the investigators (recruiters, acupuncturists, and outcome assessors) processed unified training before participating in the research for the consistency of implementation.

2.4. Sample Size. Sample size was calculated based on a study [27]; the mean weekly rates of SBMs in FC patients were 2.6 times per week with a standard deviation of 2.2 after drug treatment. Moreover, there was a 1.4-fold difference in the clinical effects between the drug and the placebo. Consequently, the desired mean defecating frequency was 4 times with a standard deviation of 3 after EA treatment. With an $\alpha$ level of 0.05 and a power of $90 \%$ [28] to detect statistically significant difference, a sample size of 213 (71 in each group) was needed and expanded to 243 (81 for each group) in consideration of a drop-out proportion of $15 \%$.

2.5. Interventions. Patients in the electroacupuncture groups received 16 sessions of acupuncture treatments: 5 times per week (once a day for 5 days continuously, followed by a 2-day interval) during the first 2 weeks and 3 times per week (once every 2-3 days) during the following 2 weeks. Each session lasted $30 \mathrm{~min}$.

LI11 and ST37 are the common used acupoints for functional gastrointestinal motility disorders [14, 17, 29-31]. In this study, acupoints of bilateral LI11 (Quchi, located at the midpoint between the lateral end of the transverse cubical crease and the lateral epicondyle of the humerus) and ST37 (Shangjuxu, located 6 cun below the lateral depression between the patellar and patellar ligament, one finger width lateral to the anterior crest of the tibia) were used.

After sterilizing the skin, acupuncture needles $(0.30 \times$ $40 \mathrm{~mm}$ or $0.30 \times 50 \mathrm{~mm}$, Human Health, Shanghai, China) were inserted into LI11 and ST37 for 15-25 mm vertically and slowly; De qi sensation (soreness, numbness, distension, and heaviness) was achieved through lifting and thrusting movements combined with twirling the needles. Then, auxiliary needles $(0.18 \times 13 \mathrm{~mm}$, Human Health, Shanghai, China) were inserted into the proximal limbs with $2 \mathrm{~mm}$ lateral to the first needle for $5 \mathrm{~mm}$ vertically, without manual stimulation. The acupuncture needle and auxiliary needle of each point were connected with an electroacupuncture instrument (HANS200E, Nanjing, Jisheng, Jiangsu, China) to form a circuit that lasted for $30 \mathrm{~min}$, with a dilatational wave at a frequency of $2 / 50 \mathrm{~Hz}$. For the LCI group, the current applied was relatively weak but can be clearly perceived by the participants. For the HCI group, the current was strong enough to reach the patients' tolerance threshold value.

Patients in mosapride control group were orally given $5 \mathrm{mg}$ mosapride citrate tablet (Dainippon Sumitomo pharmaceutical Co. Ltd., Japan) 3 times daily for 4 continuous weeks if no severe adverse events were detected.

2.6. Assessments. The primary outcome was defined as both three or more SBMs per week and an increase of one or more SBMs per week from baseline for 3 or more weeks during 4week treatment period [25]. Secondary outcomes included the change from baseline of mean stool frequency (weekly rates of SBMs from week 1 to week 8), stool consistency, and severity of straining during the 9 weeks of the study. A number of additional outcomes were assessed, including the proportion of patients who belong to severe constipation (defined as weekly SBMs less than 2 times per week [27]), the strength of association between baseline values and the presence of primary outcome and weekly SBMs $\geq 3$ dichotomized as present/absent, and the validated Patient Assessment of Constipation Quality of Life (PAC-QOL) [32]. The PAC-QOL was assessed at baseline, weeks 2, 4, and 8, with lower scores indicating a better quality of life. Adverse events were also assessed.

2.7. Statistical Analysis. SAS statistical package program (ver. 9.2, SAS Institute, Cary NC, USA) was used. All $p$ values were based on two-sided tests; $p<0.05$ was considered to be a statistically significant difference. Statistical analysis of our crowd included full analysis set (FAS, the main set of therapeutic evaluation and analysis) and safety set (SS, the main set of safety evaluation). Efficacy analysis was based on an intent-to-treat population. Continuous variables were presented as mean \pm SD (standard deviation) or mean (95\% confidence interval [CI]); categorical variables were expressed by frequency and percentage unless stated otherwise.

Categorical variables were analyzed with the used of the Cochran-Mantel-Haenszel- $\chi^{2}$ test $\left(\mathrm{CMH}-\chi^{2}\right)$. Continuous variables comparison of baseline period among the three groups was analyzed with the analysis of variance (ANOVA). And an analysis of covariance (ANCOVA) with fixed-effect terms for study group and center and with the corresponding baseline value as a covariate was used for the comparison of treatment and follow-up periods among the three groups. Finally, we used least significant difference (LSD) for further pairwise comparison if there was statistic significance of difference.

Logistic regression analysis was used to estimate the strength of association between belonging to severe constipation and the presence of primary outcome and weekly SBMs $\geq 3$ dichotomized as present/absent. The total number of SBMs was summed and divided by 4 (the number of weeks of treatment and follow-up). The data were also summarized for each of the two 2-week periods of the 4week treatment study. Two models were examined for two different independent variables: model 1 adjusted for primary outcome and model 2 adjusted for weekly SBMs $\geq 3$. Age, sex, body mass index (BMI), severe constipation, duration of constipation, group status, occupation, and education were included as covariables. We also calculated odds ratios (ORs) and $95 \%$ confidence intervals (CIs).

Patients were assumed not to have had bowel movements or to have taken rescue medications if the corresponding daily question was not answered. We used last observation carried forward (LOCF) for the missing data of the primary outcome and the secondary outcomes. 
TABLE 1: Demographic and baseline characteristics of the patients (intention-to-treat population).

\begin{tabular}{|c|c|c|c|c|}
\hline Characteristic & LCI $(n=58)$ & $\mathrm{HCI}(n=65)$ & $\mathrm{MC}(n=67)$ & $p$ value \\
\hline \multicolumn{5}{|l|}{ Sex, $n(\%)$} \\
\hline Female & $52(89.66)$ & $60(92.31)$ & $57(85.07)$ & 0.41 \\
\hline Age, years, mean $\pm S D$ & $34.00 \pm 15.62$ & $37.20 \pm 18.19$ & $43.60 \pm 17.90$ & 0.55 \\
\hline Range & $20.00-63.00$ & $22.00-62.00$ & $23.00-69.00$ & \\
\hline $\mathrm{BMI}$, mean $\pm \mathrm{SD}$ & $20.95 \pm 2.36$ & $21.22 \pm 2.98$ & $21.10 \pm 2.18$ & 0.83 \\
\hline Education, $n(\%)$ & & & & 0.09 \\
\hline Postgraduate & $8(13.79)$ & $15(23.08)$ & $21(31.34)$ & \\
\hline Bachelor & $31(53.45)$ & $26(40.00)$ & $21(31.34)$ & \\
\hline Junior college & $4(6.90)$ & $9(13.85)$ & $7(10.45)$ & \\
\hline Senior middle school & $15(25.86)$ & $13(20.00)$ & $15(22.39)$ & \\
\hline Junior middle school & $0(0.00)$ & $2(3.08)$ & $3(4.48)$ & \\
\hline $\begin{array}{l}\text { Duration of constipation, } \\
\text { months, mean } \pm S D\end{array}$ & $70.44 \pm 85.53$ & $86.29 \pm 104.06$ & $68.09 \pm 74.13$ & 0.45 \\
\hline Severe constipation, $n(\%)$ & $34(58.62)$ & $32(49.23)$ & $34(50.75)$ & 0.54 \\
\hline
\end{tabular}

The analysis of variance (ANOVA) for continuous variables and the Cochran-Mantel-Haenszel- $\chi^{2}\left(\mathrm{CMH}-\chi^{2}\right)$ test for categorical variables. $p$ values of the comparison among the three groups. Severe constipation defined as spontaneous bowel movements less than 2 times per week.

\section{Results}

3.1. Outcomes. Of the 201 patients who signed consent forms, 190 were randomly assigned to the three groups and received respective therapies (more information is detailed in Figure 1). Baseline demographic and clinical characteristics of patients in the three groups are detailed in Table 1.

3.1.1. Primary Outcome. Among the three groups, respectively, $53.45 \%, 66.15 \%$, and $52.24 \%$ of the patients in LCI, $\mathrm{HCI}$, and mosapride groups reached the primary outcome ( $p>0.05$, among the three groups) (Figure 2).

3.1.2. Secondary Outcomes. The EA groups and MC group had significant improvements compared with baseline period, including the mean SBMs/week from week 1 to week 8 (Figure 3), the stool consistency, and severity of straining at weeks 2, 4, and 8 (Table 2).

3.1.3. Additional Outcomes. The EA groups and MC group both reduced the proportion of severe constipation compared with baseline at weeks 2, 4, and 8, respectively. Moreover, at week 8 , the proportion of severe constipation in HCL was remarkably less than LCI and MC ( $p<0.05$, both) (Table 3$)$.

More patients who belong to severe constipation fulfilled the outcomes than those who were not belonging to severe constipation, including the primary outcome and the weekly SBMs $\geq 3$ in the EA groups and mosapride $(p<0.05$, all), except in mosapride group, with adjusted OR 1.42 and 95\% CI $0.45-4.47$ when analyzing the primary outcome (Table 4). In PAC-QOL, the EA groups and MC group had significant improvements on all subscales at weeks 2,4 , and 8 , respectively, compared with baseline period $(p<0.0001$, all) (Table 5).
3.2. Adverse Events. The total proportion of adverse events was $2.11 \%(4 / 190)$ in our trial. No adverse events were found in the LCI and HCI groups. In the mosapride group, 1 patient $(1.49 \%, 1 / 67)$ reported diarrhea, 2 patients $(2.99 \%, 2 / 67)$ experienced stomachache, and 1 patient $(1.49 \%, 1 / 67)$ had upper respiratory infection. The difference was significant between the electroacupuncture groups and mosapride ( $p=$ 0.0143 , among the three groups).

\section{Discussion}

The results of the 9-week multicenter, randomized, controlled trial show that all the LCI, HCI, and MC groups significantly increased the proportion of patients who fulfilled the primary outcome of three or more SBMs per week, with an increase from baseline of at least one SBM per week for 3 or more weeks of the 4 -week treatment period. The outcome is rigorous, requiring a normal bowel function of patients (since $\geq 3$ weekly SBMs are considered the low end of the range that defines normal bowel function [27]) and a sustainability of at least $75 \%$ of the treatment period [25]. Weekly SBMs are an important characteristic of bowel function, because reduced defecation frequency is typical for functional constipation [33]. The relief of functional constipation can be accurately and objectively reflected through the change of weekly SBMs [18]. Although this primary outcome was rigor, $66.15 \%$ of the patients who received high current intensity treatment still fulfilled the outcome, while $53.45 \%$ and $52.24 \%$ patients, respectively, in the LCI and MC were considered to have had a response.

Mosapride is a $5-\mathrm{HT}_{4}$ receptor agonist and can stimulate upper gastrointestinal movement [34], accelerate gastric emptying [35] and colonic motility [36,37], and improve stool frequency and consistency, especially in patients with irritable bowel syndrome with constipation (IBS-C) [38] and/or 


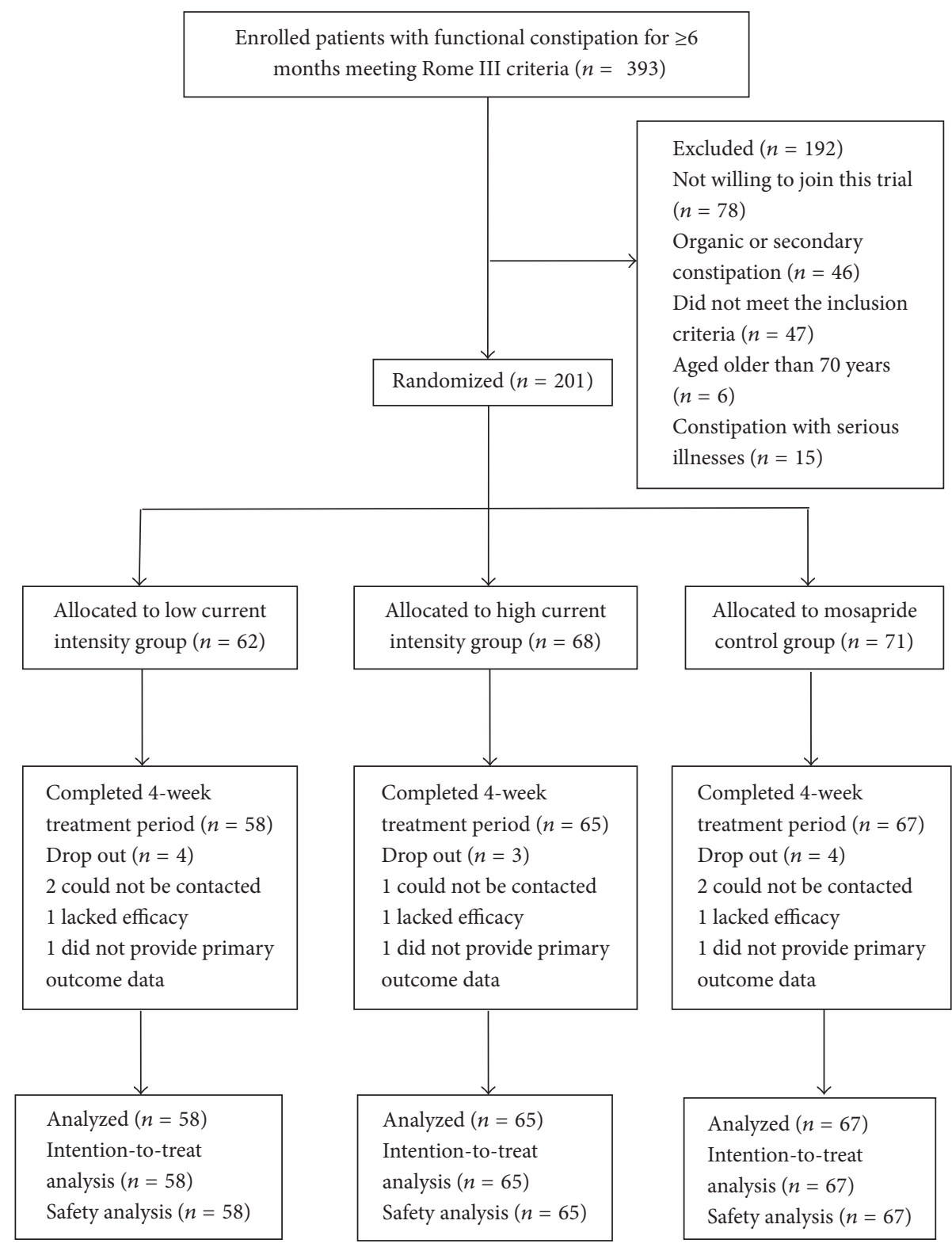

FIGURE 1: Flow chart of study participants.

functional constipation [37]. Consequently, the effects of EA during the treatment and follow-up periods can be more accurately revealed by using mosapride as the controlled intervention.

Besides improving weekly SBMs, the EA groups significantly improved stool consistency and reduced straining severity, which is in accordance with previous studies $[14,17$, $18,39,40]$. And the effects of EA on symptoms of constipation were sustained through 8 weeks, including the treatment and follow-up periods. The secondary outcomes supported the findings of the primary outcome.

The LCI and HCI significantly reduced the proportion of severe constipation patients; this is consistent with a previous study [41]. Patients with severe constipation are associated with a significantly greater risk of developing colorectal cancer and benign colorectal neoplasms than constipationfree patients [42]. Therefore, the more than $50 \%$ reduction was considered to be clinically meaningful. Moreover, at week 8, proportion of severe patients in HCI less than LCI and $\mathrm{MC}$ was detected; EA treatment was superior to that of mosapride at follow-up period, suggesting that the EA treatment had better sustained effects than mosapride. Effects of acupuncture for constipation could last for 24 weeks [43], but pharmaceuticals, such as osmotic laxatives [18] and prokinetic agents [44], are generally thought to lack sustained effects.

Severe constipation patients can better improve the weekly SBMs than those patients who had no severe constipation. This is similar to a previous study [24] that medication treatment can better improve the baseline abdominal 
TABLE 2: Secondary outcomes.

\begin{tabular}{|c|c|c|c|c|}
\hline & \multicolumn{4}{|c|}{ Mean $(95 \%$ CI $)$} \\
\hline & $\mathrm{LCI}(n=58)$ & $\mathrm{HCI}(n=65)$ & $\mathrm{MC}(n=67)$ & $p$ value \\
\hline \multicolumn{5}{|l|}{ Weekly SBMs } \\
\hline Baseline & $2.85(2.32,3.38)$ & $2.81(2.37,3.25)$ & $2.84(2.38,3.30)$ & 0.98 \\
\hline Week 2 change from baseline & $1.53(1.03,2.03)^{* * *}$ & $1.95(1.49,2.41)^{* * *}$ & $1.63(1.17,2.09)^{* * *}$ & 0.58 \\
\hline Week 4 change from baseline & $1.55(0.93,2.17)^{* * *}$ & $2.24(1.81,2.67)^{* * *}$ & $1.81(1.37,2.25)^{* * *}$ & 0.21 \\
\hline Week 8 change from baseline & $1.38(0.74,2.02)^{* *}$ & $2.02(1.62,2.42)^{* * *}$ & $1.62(1.14,2.10)^{* * *}$ & 0.19 \\
\hline \multicolumn{5}{|l|}{ Stool consistency } \\
\hline Baseline & $2.40(2.12,2.68)$ & $2.46(2.21,2.71)$ & $2.28(2.01,2.55)$ & 0.57 \\
\hline Week 2 change from baseline & $0.64(0.38,0.90)^{* * *}$ & $0.97(0.66,1.28)^{* * *}$ & $0.92(0.66,1.18)^{* * *}$ & 0.27 \\
\hline Week 4 change from baseline & $0.78(0.45,1.11)^{* * *}$ & $0.80(0.58,1.02)^{* * *}$ & $0.95(0.64,1.26)^{* * *}$ & 0.93 \\
\hline Week 8 change from baseline & $0.99(0.73,1.25)^{* * *}$ & $0.79(0.53,1.05)^{* * *}$ & $0.85(0.57,1.13)^{* * *}$ & 0.73 \\
\hline \multicolumn{5}{|l|}{ Straining severity } \\
\hline Baseline & $1.20(1.04,1.36)$ & $1.33(1.19,1.47)$ & $1.23(1.07,1.39)$ & 0.54 \\
\hline Week 2 change from baseline & $-0.50(-0.67,-0.33)^{* * *}$ & $-0.49(-0.65,-0.33)^{* * *}$ & $-0.33(-0.49,-0.17)^{* *}$ & 0.14 \\
\hline Week 4 change from baseline & $-0.44(-0.62,-0.26)^{* * *}$ & $-0.53(-0.71,-0.35)^{* * *}$ & $-0.41(-0.60,-0.22)^{* *}$ & 0.38 \\
\hline Week 8 change from baseline & $-0.54(-0.77,-0.31)^{* * *}$ & $-0.59(-0.76,-0.42)^{* * *}$ & $-0.34(-0.52,-0.16)^{* *}$ & 0.20 \\
\hline
\end{tabular}

*** $p<0.0001$ versus baseline; ${ }^{* *} p<0.001$ versus baseline.

${ }^{* * *} p$ and ${ }^{* *} p$ values were used through the Signed-Rank test.

$p$ values were for the comparison among the three groups and were calculated with the analysis of covariance (ANCOVA), except for the comparison of the baseline values, which used an analysis of variance (ANOVA).

(1) SBMs denote spontaneous bowel movements.

(2) Stool consistency was assessed with the use of the 7-point Bristol Stool Form Scale (BSFS): 1 indicating separate, hard lumps, like nuts (hard to pass); 2 sausage-shaped but lumpy; 3 like a sausage but with cracks on the surface; 4 like a sausage or snake, smooth, and soft; 5 soft blobs with clear-cut edges (passed easily); 6 fluffy pieces with ragged edges or a mushy stool; and 7 watery, not solid pieces (entirely liquid).

(3) Straining severity was assessed by means of a 4-point ordinal scale with the following responses, while 0 indicates not at all, 1 a little bit, 2 a moderate amount, and 3 a great deal and an extreme amount.

TABLE 3: The proportion of severe constipation patients in LCI, HCI, and mosapride groups.

\begin{tabular}{|c|c|c|c|c|}
\hline Severe constipation, $n(\%)$ & LCI $(n=58)$ & $\mathrm{HCI}(n=65)$ & $\mathrm{MC}(n=67)$ & $p$ value \\
\hline Baseline & $34(58.62)$ & $32(49.23)$ & $34(50.75)$ & 0.54 \\
\hline $2 \mathrm{~W}$ & $20(34.48)^{*}$ & $12(18.46)^{* *}$ & $17(25.37)^{*}$ & 0.13 \\
\hline $4 \mathrm{~W}$ & $15(25.86)^{* *}$ & $12(18.46)^{* *}$ & $17(25.37)^{*}$ & 0.54 \\
\hline $8 \mathrm{~W}$ & $15(25.86)^{* *}$ & $6(9.23)^{* * 9}$ & $17(25.37)^{*}$ & 0.03 \\
\hline
\end{tabular}

${ }^{* *} p<0.001$, versus baseline; ${ }^{*} p<0.01$, versus baseline. ${ }^{9} p<0.05$, versus LCI; ${ }^{\#} p<0.05$, versus mosapride.

Severe constipation defined as spontaneous bowel movements less than 2 times per week.

* $p$ and ${ }^{* *} p$ values were used through the Signed-Rank test.

" $p$ and $p$ values were used through the least significant difference (LSD).

$p$ values were for the comparison among the three groups and were calculated with the analysis of covariance (ANCOVA), except for the comparison of the baseline values, which used an analysis of variance (ANOVA).

TABLE 4: Primary outcome of and weekly SBMs $\geq 3$ among patients belonging to severe constipation adjusted for baseline data.

\begin{tabular}{lccr}
\hline & & Adjusted OR $(95 \%$ CI $)$ \\
& LCI $(n=58)$ & HCI $(n=65)$ & $1.42(0.45,4.47)$ \\
Primary outcome & $3.48(1.06,11.38)^{*}$ & $3.67(1.01,13.27)^{*}$ & \\
Weekly SBMs $\geq 3$ & & & $12.15(2.18,67.61)^{* *}$ \\
Weeks 1-2 & $21.13(3.26,137.13)^{* *}$ & $17.51(2.40,127.69)^{* *}$ & $8.81(2.17,35.77)^{* *}$ \\
Weeks 3-4 & $30.97(3.31,289.53)^{* *}$ & $29.31(3.06,281.29)^{* *}$ & $7.55(1.76,32.40)^{* *}$ \\
Weeks 1-4 & $29.29(3.54,242.46)^{* *}$ & $40.30(3.55,457.07)^{* *}$ & $4.04(1.15,14.16)^{*}$ \\
Weeks 5-8 & $29.60(3.85,227.92)^{* *}$ & & $46.81)^{* *}$ \\
\hline
\end{tabular}

$\mathrm{CI}=$ confidence interval; $\mathrm{OR}=$ odds ratio; ${ }^{*} p<0.05 ;{ }^{* *} p<0.01$.

Severe constipation defined as spontaneous bowel movements less than 2 times per week.

Variables included in the model: age, sex, body mass index (BMI), severe constipation, duration of constipation, group status, occupation, and education. 
TABLE 5: PAC-QOL questionnaire.

\begin{tabular}{|c|c|c|c|c|}
\hline & \multicolumn{4}{|c|}{ Mean $(95 \% \mathrm{CI})$} \\
\hline & LCI $(n=58)$ & HCI $(n=65)$ & $\mathrm{MC}(n=67)$ & $p$ value \\
\hline \multicolumn{5}{|l|}{ Overall scores } \\
\hline Week 2 change from baseline & $0.67(0.55,0.79)^{* * * \S}$ & $0.63(0.49,0.77)^{* * * \#}$ & $0.40(0.26,0.54)^{* * *}$ & 0.00 \\
\hline Week 4 change from baseline & $0.72(0.59,0.85)^{* * *}$ & $0.82(0.68,0.96)^{* * * \#}$ & $0.47(0.35,0.59)^{* * *}$ & 0.00 \\
\hline Week 8 change from baseline & $0.78(0.64,0.92)^{* * *}$ & $0.93(0.77,1.09)^{* * * \#}$ & $0.56(0.42,0.70)^{* * *}$ & 0.00 \\
\hline \multicolumn{5}{|l|}{ Physical discomfort } \\
\hline Week 2 change from baseline & $0.74(0.56,0.92)^{* * *}$ & $0.67(0.49,0.85)^{* * *}$ & $0.53(0.34,0.72)^{* * *}$ & 0.26 \\
\hline Week 4 change from baseline & $0.88(0.72,1.04)^{* * *}$ & $0.92(0.74,1.10)^{* * * \#}$ & $0.56(0.39,0.73)^{* * *}$ & 0.01 \\
\hline Week 8 change from baseline & $0.91(0.72,1.10)^{* * *}$ & $1.08(0.90,1.26)^{* * *}$ & $0.72(0.53,0.91)^{* * *}$ & 0.06 \\
\hline \multicolumn{5}{|l|}{ Psychosocial discomfort } \\
\hline Week 2 change from baseline & $0.44(0.31,0.57)^{* * *}$ & $0.34(0.23,0.45)^{* * *}$ & $0.27(0.12,0.42)^{* * *}$ & 0.12 \\
\hline Week 4 change from baseline & $0.47(0.33,0.61)^{* * *}$ & $0.49(0.33,0.65)^{* * *}$ & $0.32(0.18,0.46)^{* * *}$ & 0.10 \\
\hline Week 8 change from baseline & $0.57(0.43,0.71)^{* * *}$ & $0.63(0.43,0.83)^{* * *}$ & $0.44(0.28,0.60)^{* * *}$ & 0.22 \\
\hline \multicolumn{5}{|l|}{ Worries and concerns } \\
\hline Week 2 change from baseline & $0.71(0.57,0.85)^{* * * \S}$ & $0.63(0.46,0.80)^{* * * \#}$ & $0.42(0.26,0.58)^{* * *}$ & 0.02 \\
\hline Week 4 change from baseline & $0.75(0.59,0.91)^{* * *}$ & $0.86(0.69,1.03)^{* * * \#}$ & $0.52(0.37,0.67)^{* * *}$ & 0.01 \\
\hline Week 8 change from baseline & $0.79(0.60,0.98)^{* * *}$ & $0.99(0.81,1.17)^{* * * \# 9}$ & $0.60(0.43,0.77)^{* * *}$ & 0.01 \\
\hline \multicolumn{5}{|l|}{ Satisfaction } \\
\hline Week 2 change from baseline & $0.92(0.67,1.17)^{* * *}$ & $1.02(0.78,1.26)^{* * * \#}$ & $0.45(0.26,0.64)^{* * *}$ & 0.01 \\
\hline Week 4 change from baseline & $0.93(0.69,1.17)^{* * *}$ & $1.15(0.89,1.41)^{* * * \# 9}$ & $0.52(0.33,0.71)^{* * *}$ & 0.00 \\
\hline Week 8 change from baseline & $1.00(0.78,1.22)^{* * *}$ & $1.18(0.91,1.45)^{* * * \#}$ & $0.54(0.30,0.78)^{* * *}$ & 0.00 \\
\hline
\end{tabular}

${ }^{* * * *} p<0.0001$, versus baseline; ${ }^{*} p<0.05$, versus mosapride.

${ }^{\S} p<0.05$, versus mosapride; ${ }^{9} p<0.05$, versus LCI

${ }^{* * *} p$ values were used through the Signed-Rank test.

${ }^{\#} p,{ }^{9} p$, and ${ }^{\S} p$ values were used through the least significant difference (LSD).

$p$ values were for the comparison among the three groups and were calculated with the analysis of covariance (ANCOVA), except for the comparison of the baseline values, which used an analysis of variance (ANOVA).

symptoms in the severe subpopulations than that population, which included patients with milder baseline abdominal symptoms. The significant difference presented rising trend in HCI, while exhibiting declining trend in mosapride, which is in accordance with that fewer proportion of severe constipation patients in HCI than LCI and mosapride at follow-up period.

The EA treatment remarkably improved the diseaserelated quality of patients' life, which is consistent with a previous study [45]. The PAC-QOL is a useful constipation patient-reported outcomes assessment tool, measuring patient health-related quality of life and satisfaction [32]. Improvements in PAC-QOL overall score and satisfaction score are associated with improvements in symptoms of chronic constipation [46]. Our data suggested that HCI group showed greater improvement than mosapride on satisfaction and worries and concerns subscales; moreover, HCI was better than LCI in improving level of satisfaction at week 4 and worries and concerns condition at week 8 . It indicates that EA treatment is superior to that of mosapride in improving the patients' life quality and the satisfaction level of treatment.

In our trial, the total proportion of adverse events was $2.11 \%$, only 4 patients from the mosapride group had adverse events, and no adverse events were found in the LCI and HCI groups. EA or acupuncture had no serious adverse events reported in treatment of FC [17] or IBS [47]. Acupuncture had less emergency drug usage and side effects than lactulose in the treatment of FC [18]. EA treatment is safer than mosapride for FC. The occurrence of adverse events in

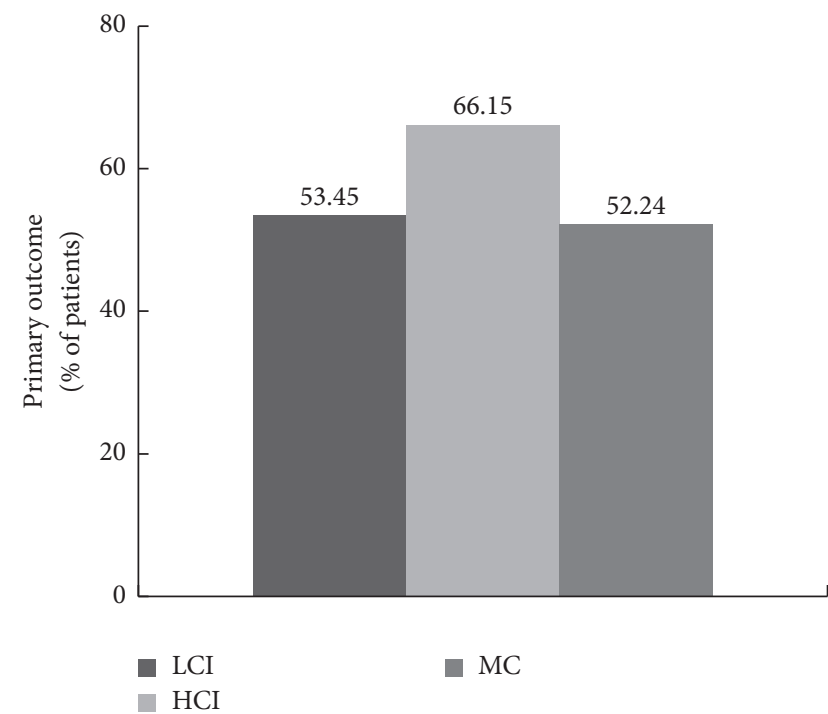

FIgURE 2: The primary outcome in the LCI, HCI, and mosapride groups. The primary outcome was defined as a weekly frequency of three or more spontaneous bowel movements (SBMs) and an increase of one or more SBMs from baseline for at least 3 weeks of the 4 -week treatment period. The Cochran-Mantel-Haenszel- $\chi^{2}$ $\left(\mathrm{CMH}-\chi^{2}\right)$ test was used.

mosapride is not surprising, since mosapride is well tolerated, with diarrhea, loose stools, dry mouth, malaise, and headache being reported in $<5 \%$ of patients [ 48 ]. Thus, the patients who 


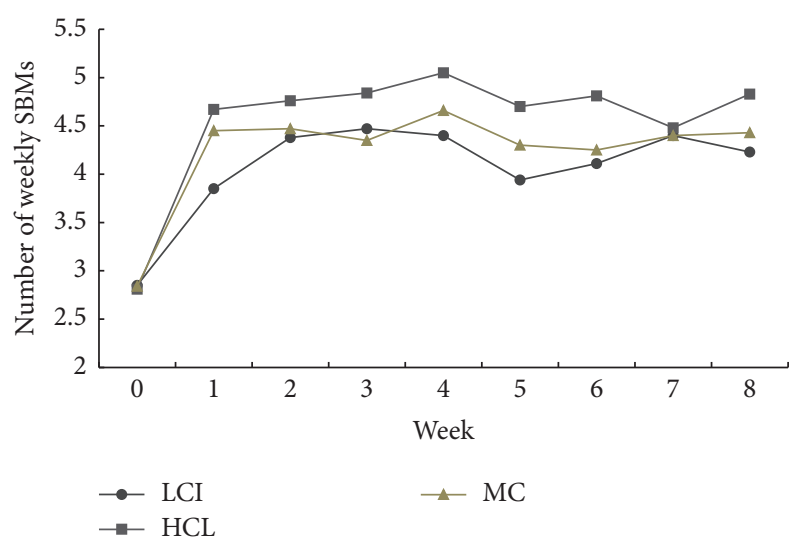

FIGURE 3: Mean number of weekly spontaneous bowel movements. The LCI, HCI, and mosapride resulted in a significant increase in the number of weekly SBMs, as compared with baseline period at each time frame from week 1 to week 8 , respectively $(p<0.0001$, all, while in LCI, at week 5 and week $8, p=0.001$, and $p=0.0001$, resp.). Signed-Rank test was used.

are not willing to take pharmacologic treatment and/or have contraindications to agents can choose acupuncture for their constipation symptoms.

Our data suggest that EA treatment is effective and safe at both current intensities in the improvement of functional constipation. There is no significant difference between LCI and HCI in intestinal function except for proportion of severe constipation patients at week 8 . A previous study [18] also indicated that deep needing and shallow needing both improve the symptoms of FC. It has long been known that acupuncture is an individualized treatment rather than a standardized needle manipulation [49]; the defined low and high intensity in the current study are according to the patients experience. Moreover, in the LCI and HCI, all patients achieved the De qi sensation; De qi is the sine qua non of acupuncture for the achievement of a clinical therapeutic effect according to TCM [50]. And EA stimulation can strengthen the De qi sensation. However, sufficient evidence with large sample size and long treatment and follow-up period studies to prove the connection of the current intensity parameter and the therapeutic effects of EA is needed.

The limitations of this clinical trial should be addressed. First, only different current intensities of EA were compared, no placebo control or no sham acupuncture control; it might not be accurate enough to reflect the therapeutic effects of EA. An inadequate number of patients to prove the efficacy were another limitation; possible reasons are as follows: (i) we had only three hospitals to help us recruit FC patients; (ii) some patients with lighter symptoms who did not care about their problems might pay little attentions to the information from our hospitals; (iii) some other patients had insufficient time to participate in our clinical trial. Besides, our 1-week screening period is slightly short, and our 4-week treatment period and 4-week follow-up period might not be long enough for the interventions to show completely the effects of EA. Patients might have taken laxatives or stool softeners before study enrollment; the 1-week screening period might not accurately reflect the severity of constipation patients. Acupuncture had better sustained effects than pharmaceuticals [18]; therefore, the follow-up period should be long enough that can accurately reflect the sustained effects of acupuncture. More rigorous and high-quality studies with larger sample sizes are required.

In conclusion, the EA treatment is effective and safe at both current intensities for patients with functional constipation. And therapeutic effects of low and high current intensity are not superior to that of mosapride in improving the weekly SBMs, stool consistency, and straining severity. However, EA treatment is superior to that of mosapride in improving patients' life quality and satisfaction level of treatment. Meanwhile, EA treatment has fewer adverse events compared with mosapride. These findings might qualify the superiority of EA.

\section{Competing Interests}

All authors declared that they have no competing interests.

\section{Authors' Contributions}

Xiao $\mathrm{Wu}$ wrote the initial draft of the manuscript, enrolled patients in the study, and assisted in the interpretation of data. Guangying Huang designed the study. Cuihong Zheng, Mingmin Zhang, Wei Wang, Shabei Xu, and Minjie Xie contributed to the manuscript development. Xiaohu Xu, Pei Ding, Haoxu Dong, Fan Xiong, Man Tian, and Ying Wang enrolled patients in the study. All authors approved the final version of the manuscript.

\section{Acknowledgments}

This study was supported by grants from the National Basic Research Program (no. 2011CB505203).

\section{References}

[1] D. A. Drossman, "The functional gastrointestinal disorders and the Rome III process," Gastroenterology, vol. 130, no. 5, pp. 13771390, 2006.

[2] A. E. Bharucha, S. D. Dorn, A. Lembo, and A. Pressman, "American gastroenterological association medical position statement on constipation," Gastroenterology, vol. 144, no. 1, pp. 211-217, 2013.

[3] C. Dennison, M. Prasad, A. Lloyd, S. K. Bhattacharyya, R. Dhawan, and K. Coyne, "The health-related quality of life and economic burden of constipation," PharmacoEconomics, vol. 23, no. 5, pp. 461-476, 2005.

[4] J. F. Johanson and J. Kralstein, "Chronic constipation: a survey of the patient perspective," Alimentary Pharmacology and Therapeutics, vol. 25, no. 5, pp. 599-608, 2007.

[5] N. C. Suares and A. C. Ford, "Prevalence of, and risk factors for, chronic idiopathic constipation in the community: systematic review and meta-analysis," American Journal of Gastroenterology, vol. 106, no. 9, pp. 1582-1591, 2011.

[6] R. Park, S. Mikami, J. Leclair et al., "Inpatient burden of childhood functional GI disorders in the USA: an analysis of national 
trends in the USA from 1997 to 2009," Neurogastroenterology and Motility, vol. 27, no. 5, pp. 684-692, 2015.

[7] T. Sommers, C. Corban, N. Sengupta et al., "Emergency department burden of constipation in the United States from 2006 to 2011," The American Journal of Gastroenterology, vol. 110, no. 4, pp. 572-579, 2015.

[8] S. Müller-Lissner, J. Tack, Y. Feng, F. Schenck, and R. Specht Gryp, "Levels of satisfaction with current chronic constipation treatment options in Europe-an internet survey," Alimentary Pharmacology and Therapeutics, vol. 37, no. 1, pp. 137-145, 2013.

[9] Z. C. Yang, S.-H. Yang, S.-S. Yang, and D.-S. Chen, "A hospitalbased study on the use of alternative medicine in patients with chronic liver and gastrointestinal diseases," American Journal of Chinese Medicine, vol. 30, no. 4, pp. 637-643, 2002.

[10] T. J. Kaptchuk, "Acupuncture: theory, efficacy, and practice," Annals of Internal Medicine, vol. 136, no. 5, pp. 374-383, 2002.

[11] M. Iwa, M. Matsushima, Y. Nakade, T. N. Pappas, M. Fujimiya, and T. Takahashi, "Electroacupuncture at ST-36 accelerates colonic motility and transit in freely moving conscious rats," American Journal of Physiology-Gastrointestinal and Liver Physiology, vol. 290, no. 2, pp. G285-G292, 2006.

[12] J. J. Y. Sung, "Acupuncture for gastrointestinal disorders: myth or magic," Gut, vol. 51, no. 5, pp. 617-619, 2002.

[13] J. Yin and J. D. Z. Chen, "Gastrointestinal motility disorders and acupuncture," Autonomic Neuroscience: Basic and Clinical, vol. 157, no. 1-2, pp. 31-37, 2010.

[14] X. Wang and J. Yin, "Complementary and alternative therapies for chronic constipation," Evidence-Based Complementary and Alternative Medicine, vol. 2015, Article ID 396396, 11 pages, 2015.

[15] C. Zhang, L. Guo, X. Guo, G. Li, and X. Guo, "Short and long-term efficacy of combining Fuzhengliqi mixture with acupuncture in treatment of functional constipation," Journal of Traditional Chinese Medicine, vol. 33, no. 1, pp. 51-59, 2013.

[16] L.-K. Guo, C.-X. Zhang, and X.-F. Guo, "Acupuncture combined with Chinese herbal medicine plantain and Senna Granule in treatment of functional constipation: a randomized, controlled trial," Zhong Xi Yi Jie He Xue Bao, vol. 9, no. 11, pp. 1206-1214, 2011.

[17] N. Da, X. Wang, H. Liu et al., "The effectiveness of electroacupuncture for functional constipation: a randomized, controlled, clinical trial," Evidence-Based Complementary and Alternative Medicine, vol. 2015, Article ID 670963, 5 pages, 2015.

[18] J. Wu, B. Liu, N. Li et al., "Effect and safety of deep needling and shallow needling for functional constipation: a multicenter, randomized controlled trial," Medicine, vol. 93, no. 28, p. e284, 2014.

[19] B. Chen, S.-X. Hu, B.-H. Liu et al., "Efficacy and safety of electroacupuncture with different acupoints for chemotherapyinduced nausea and vomiting: study protocol for a randomized controlled trial," Trials, vol. 16, no. 1, article no. 212, 2015.

[20] Z. Liu, J. Liu, Y. Zhao et al., "The efficacy and safety study of electro-acupuncture for severe chronic functional constipation: study protocol for a multicenter, randomized, controlled trial," Trials, vol. 14, no. 1, article no. 176, 2013.

[21] C. H. Zheng, G. Y. Huang, X. H. Xu et al., "Electro-acupuncture with different current intensities to treat functional constipation: a study protocol for a randomized controlled trial," Trials, vol. 14, no. 1, article no. 344, 2013.

[22] "Rome III Diagnostic Criteria for Functional Gastrointestinal Disorders," http://www.romecriteria.org/assets/pdf/19_RomeIII_ apA.
[23] S. Rao, A. J. Lembo, S. J. Shiff et al., "A 12-week, randomized, controlled trial with a 4-week randomized withdrawal period to evaluate the efficacy and safety of linaclotide in irritable bowel syndrome with constipation," American Journal of Gastroenterology, vol. 107, no. 11, pp. 1714-1724, 2012.

[24] S. S. C. Rao, E. M. M. Quigley, S. J. Shiff et al., "Effect of linaclotide on severe abdominal symptoms in patients with irritable bowel syndrome with constipation," Clinical Gastroenterology and Hepatology, vol. 12, no. 4, pp. 616-623, 2014.

[25] A. J. Lembo, H. A. Schneier, S. J. Shiff et al., "Two randomized trials of linaclotide for chronic constipation," New England Journal of Medicine, vol. 365, no. 6, pp. 527-536, 2011.

[26] S. J. Lewis and K. W. Heaton, "Stool form scale as a useful guide to intestinal transit time," Scandinavian Journal of Gastroenterology, vol. 32, no. 9, pp. 920-924, 1997.

[27] M. Camilleri, R. Kerstens, A. Rykx, and L. Vandeplassche, "A placebo-controlled trial of prucalopride for severe chronic constipation," New England Journal of Medicine, vol. 358, no. 22, pp. 2344-2354, 2008.

[28] J. Xu, L. Wang, and J. Xia, "Sample size estimation and statistical inference in a three-arm non-inferiority clinical trial including a placebo and an active control," Chinese Journal of Health Statistics, vol. 26, no. 3, pp. 255-257, 2009.

[29] X. Xu, C. Zheng, M. Zhang, W. Wang, and G. Huang, "A randomized controlled trial of acupuncture to treat functional constipation: design and protocol," BMC Complementary and Alternative Medicine, vol. 14, article no. 423, 2014.

[30] C.-Y. Chen, M.-D. Ke, C.-D. Kuo, C.-H. Huang, Y.-H. Hsueh, and J.-R. Chen, "The Influence of electro-acupuncture stimulation to female constipation patients," American Journal of Chinese Medicine, vol. 41, no. 2, pp. 301-313, 2013.

[31] X. Zhu, Z. Liu, H. Qu et al., "The effect and mechanism of electroacupuncture at LI11 and ST37 on constipation in a rat model," Acupuncture in Medicine, vol. 34, no. 3, pp. 194-200, 2016.

[32] D. Dubois, H. Gilet, M. Viala-Danten, and J. Tack, "Psychometric performance and clinical meaningfulness of the Patient Assessment of Constipation-quality of Life questionnaire in prucalopride (RESOLOR) trials for chronic constipation," Neurogastroenterology and Motility, vol. 22, no. 2, pp. e54-e63, 2010.

[33] P. E. Hyman, C. Di Lorenzo, L. L. Prestridge, N. N. Youssef, and R. Ueno, "Lubiprostone for the treatment of functional constipation in children," Journal of Pediatric Gastroenterology and Nutrition, vol. 58, no. 3, pp. 283-291, 2014.

[34] Q. Liu, C.-C. Feng, E.-M. Wang, X.-J. Yan, and S.-L. Chen, "Efficacy of mosapride plus proton pump inhibitors for treatment of gastroesophageal refux disease: a systematic review," World Journal of Gastroenterology, vol. 19, no. 47, pp. 9111-9118, 2013.

[35] Y. Mizuta, S. Shikuwa, H. Isomoto et al., "Recent insights into digestive motility in functional dyspepsia," Journal of Gastroenterology, vol. 41, no. 11, pp. 1025-1040, 2006.

[36] A. Inui, T. Yoshikawa, R. Nagai, N. Yoshida, and T. Ito, "Effects of mosapride citrate, a 5-HT4 receptor agonist, on colonic motility in conscious guinea pigs," Japanese Journal of Pharmacology, vol. 90, no. 4, pp. 313-320, 2002.

[37] M. Kanazawa, S. Watanabe, C. Tana, H. Komuro, M. Aoki, and S. Fukudo, "Effect of 5-HT4 receptor agonist mosapride citrate on rectosigmoid sensorimotor function in patients with irritable bowel syndrome," Neurogastroenterology and Motility, vol. 23, no. 8, pp. 754-e332, 2011. 
[38] C. H. Choi, J. G. Kwon, S. K. Kim et al., "Efficacy of combination therapy with probiotics and mosapride in patients with IBS without diarrhea: a randomized, double-blind, placebocontrolled, multicenter, phase II trial," Neurogastroenterology \& Motility, vol. 27, no. 5, pp. 705-716, 2015.

[39] X. Zhu, Z. Liu, H. Qu et al., "The effect and mechanism of electroacupuncture at LI11 and ST37 on constipation in a rat model," Acupuncture in Medicine, vol. 34, no. 3, pp. 194-200, 2016.

[40] Q. Zheng, H. Zheng, L. Lu et al., "Acupuncture for functional constipation: protocol of an individual patient data metaanalysis," BMJ Open, vol. 5, no. 5, Article ID e007137, 2015.

[41] J. Liu, W. Zhou, H. Lv et al., "Law of the meridian abnormality based on the effectiveness of electroacupuncture for severe functional constipation," Zhongguo Zhen Jiu, vol. 35, no. 8, pp. 785-790, 2015.

[42] A. Guérin, R. Mody, B. Fok et al., "Risk of developing colorectal cancer and benign colorectal neoplasm in patients with chronic constipation," Alimentary Pharmacology and Therapeutics, vol. 40, no. 1, pp. 83-92, 2014.

[43] W.-N. Peng, L. Wang, Z.-S. Liu et al., "Analysis on follow-up efficacy and safety of slow transit constipation treated with individualized deep puncture at Tianshu (ST 25): a multicentral randomized controlled trial," Zhongguo Zhen Jiu, vol. 33, no. 10, pp. 865-869, 2013.

[44] A. Emmanuel, M. Cools, L. Vandeplassche, and R. Kerstens, "Prucalopride improves bowel function and colonic transit time in patients with chronic constipation: an integrated analysis," American Journal of Gastroenterology, vol. 109, no. 6, pp. 887894, 2014.

[45] C. H. Lau, X. Wu, V. C. Chung et al., "Acupuncture and related therapies for symptom management in palliative cancer care: systematic review and meta-analysis," Medicine, vol. 95, no. 9, p. e2901, 2016.

[46] J. Tack, M. Camilleri, D. Dubois, L. Vandeplassche, A. Joseph, and R. Kerstens, "Association between health-related quality of life and symptoms in patients with chronic constipation: an integrated analysis of three phase 3 trials of prucalopride," Neurogastroenterology and Motility, vol. 27, no. 3, pp. 397-405, 2015.

[47] E. Manheimer, L. S. Wieland, K. Cheng et al., "Acupuncture for irritable bowel syndrome: systematic review and meta-analysis," The American Journal of Gastroenterology, vol. 107, no. 6, pp. 835-848, 2012.

[48] M. P. Curran and D. M. Robinson, "Mosapride: in gastrointestinal disorders," Drugs, vol. 68, no. 7, pp. 981-991, 2008.

[49] J. Luo, H. Xu, and B. Liu, "Real world research: a complementary method to establish the effectiveness of acupuncture," $B M C$ Complementary and Alternative Medicine, vol. 15, no. 1, article no. 153, 2015.

[50] D.-S. Tian, J. Xiong, Q. Pan et al., "De $Q i$, a threshold of the stimulus intensity, elicits the specific response of acupoints and intrinsic change of human brain to acupuncture," EvidenceBased Complementary and Alternative Medicine, vol. 2014, Article ID 914878, 11 pages, 2014. 


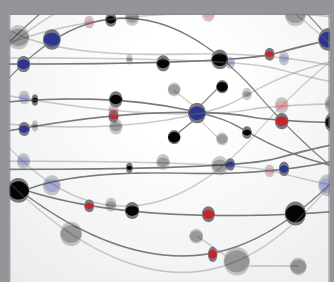

The Scientific World Journal
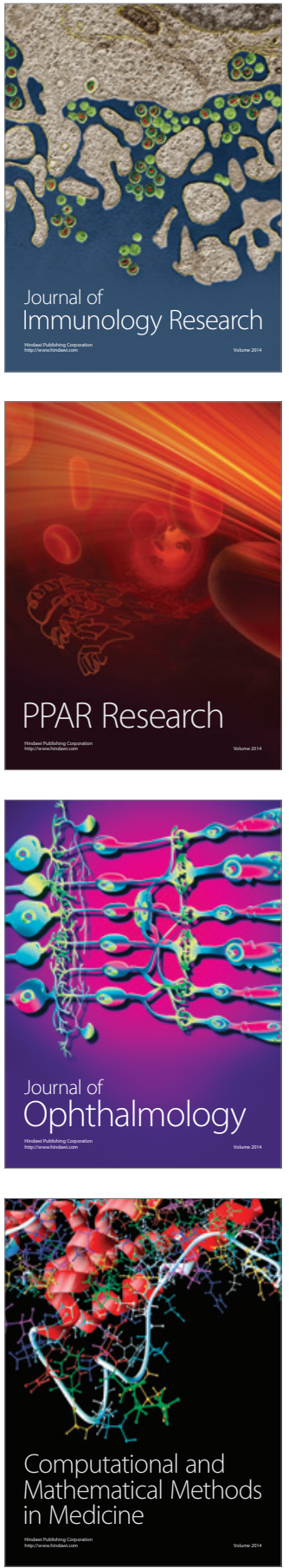

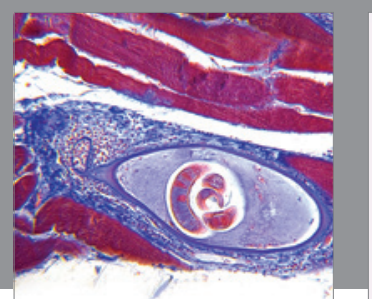

Gastroenterology Research and Practice
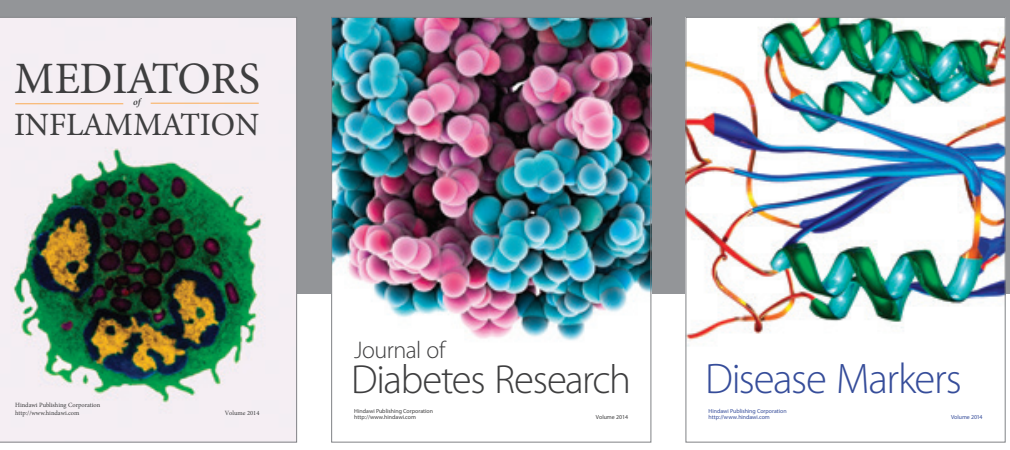

Disease Markers

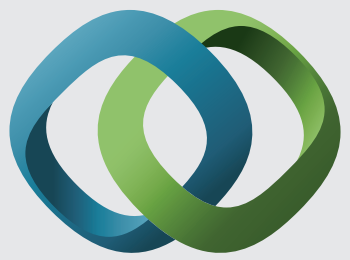

\section{Hindawi}

Submit your manuscripts at

https://www.hindawi.com
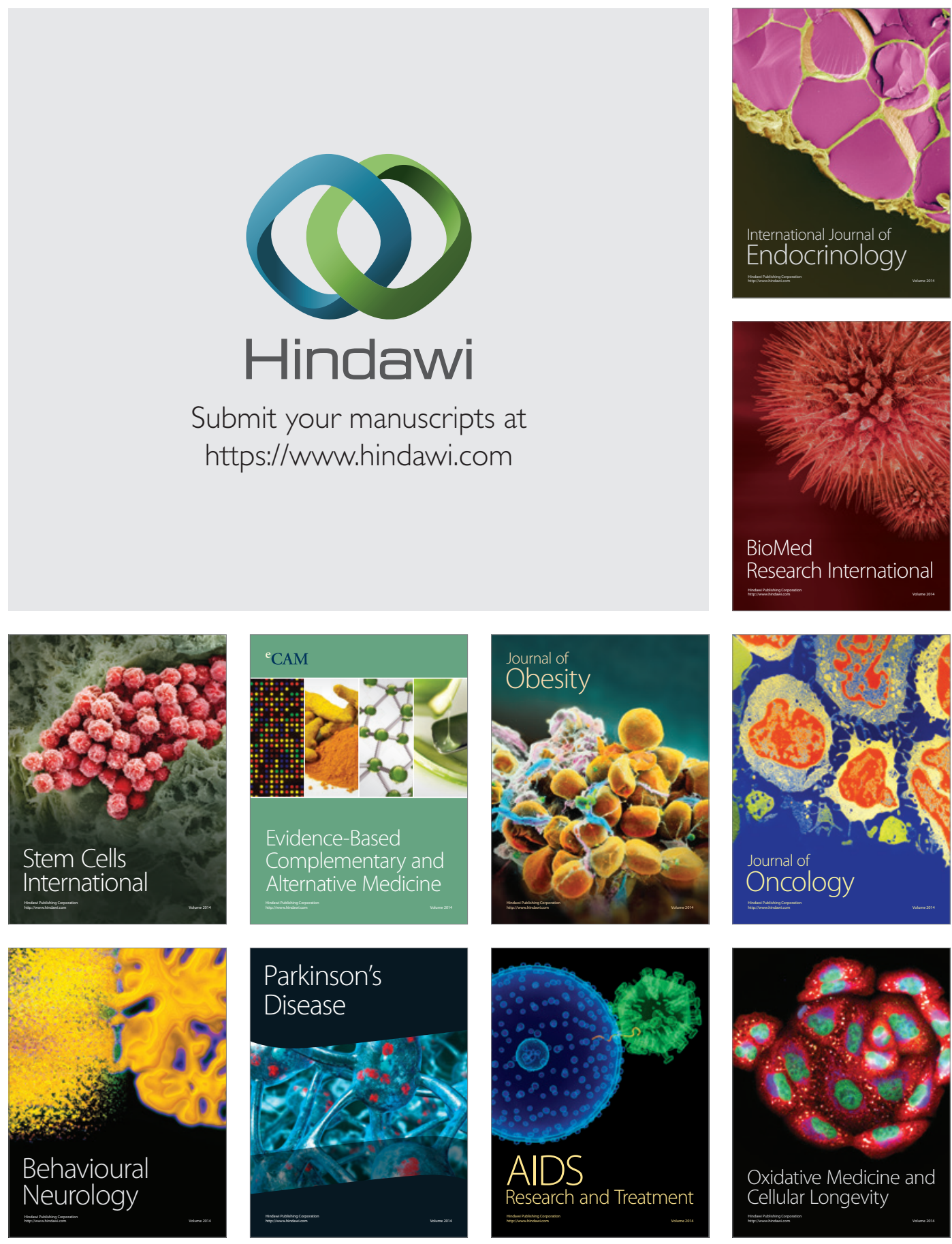